\title{
The diameter of the CoRoT target HD 49933
}

\section{Combining the 3D limb darkening, asteroseismology, and interferometry}

\author{
L. Bigot ${ }^{1}$, D. Mourard ${ }^{2}$, P. Berio ${ }^{2}$, F. Thévenin ${ }^{1}$, R. Ligi ${ }^{2}$, I. Tallon-Bosc ${ }^{3}$, O. Chesneau ${ }^{2}$, O. Delaa ${ }^{2}$, N. Nardetto ${ }^{2}$, \\ K. Perraut ${ }^{4}$, Ph. Stee ${ }^{2}$, T. Boyajian ${ }^{5}$, P. Morel $^{1}$, B. Pichon ${ }^{1}$, P. Kervella ${ }^{6}$, F. X. Schmider ${ }^{2}$, H. McAlister ${ }^{7,8}$, \\ T. ten Brummelaar ${ }^{8}$, S. T. Ridgway ${ }^{9}$, J. Sturmann ${ }^{8}$, L. Sturmann ${ }^{8}$, N. Turner ${ }^{8}$, C. Farrington ${ }^{8}$, and P. J. Goldfinger ${ }^{8}$ \\ ${ }^{1}$ Université Nice-Sophia Antipolis, Observatoire de la Côte d'Azur, CNRS UMR 6202, BP 4229, 06304 Nice Cedex, France \\ e-mail: lbigot@oca.eu \\ 2 Université Nice-Sophia Antipolis, Observatoire de la Côte d'Azur, CNRS UMR 6525, BP 4229, 06304 Nice Cedex, France \\ 3 UCBL/CNRS CRAL, 9 avenue Charles André, 69561 Saint Genis Laval Cedex, France \\ ${ }^{4}$ UJF-Grenoble 1/CNRS-INSU, Institut de Planétologie et d'Astrophysique de Grenoble, UMR 5274, 38041 Grenoble, France \\ 5 CHARA and Department of Physics and Astronomy, Georgia State University, PO Box 4106, Atlanta, GA 30302-4106, USA \\ ${ }^{6}$ LESIA, Observatoire de Paris, CNRS UMR 8109, UPMC, Université Paris Diderot, 5 place Jules Janssen, 92195 Meudon, France \\ 7 Georgia State University, PO Box 3969, Atlanta GA 30302-3969, USA \\ 8 CHARA Array, Mount Wilson Observatory, 91023 Mount Wilson CA, USA \\ 9 National Optical Astronomy Observatory, PO Box 26732, Tucson, AZ 85726, USA
}

Received 26 May 2011 / Accepted 22 August 2011

\section{ABSTRACT}

\begin{abstract}
Context. The interpretation of stellar pulsations in terms of internal structure depends on the knowledge of the fundamental stellar parameters. Long-base interferometers permit us to determine very accurate stellar radii, which are independent constraints for stellar models that help us to locate the star in the HR diagram.

Aims. Using a direct interferometric determination of the angular diameter and advanced three-dimensional (3D) modeling, we derive the radius of the CoRoT target HD 49933 and reduce the global stellar parameter space compatible with seismic data.

Methods. The VEGA/CHARA spectro-interferometer is used to measure the angular diameter of the star. A 3D radiative hydrodynamical simulation of the surface is performed to compute the limb darkening and derive a reliable diameter from visibility curves. The other fundamental stellar parameters (mass, age, and $T_{\text {eff }}$ ) are found by fitting the large and small p-mode frequency separations using a stellar evolution model that includes microscopic diffusion.

Results. We obtain a limb-darkened angular diameter of $\theta_{\mathrm{LD}}=0.445 \pm 0.012$ mas. With the Hipparcos parallax, we obtain a radius of $R=1.42 \pm 0.04 R_{\odot}$. The corresponding stellar evolution model that fits both large and small frequency separations has a mass of $1.20 \pm 0.08 M_{\odot}$ and an age of $2.7 \mathrm{~Gy}$. The atmospheric parameters are $T_{\text {eff }}=6640 \pm 100 \mathrm{~K}, \log g=4.21 \pm 0.14$, and $[\mathrm{Fe} / \mathrm{H}]=-0.38$.
\end{abstract}

Key words. stars: oscillations - hydrodynamics - stars: fundamental parameters - techniques: interferometric - asteroseismology stars: individual: HD 49933

\section{Introduction}

The F5V solar-like pulsator HD 49933 (HR 2530, HIP 32851) is one of the primary targets of the CoRoT space mission (Baglin et al. 2006). The discovery of its oscillation was made from the ground by observing highly resolved time-series spectra (Mosser et al. 2005). Since the launch of the spacecraft in December 2006, two runs of 60 and 137 days have been performed leading to a rich p-mode spectrum of 51 frequencies corresponding to the $\ell=0,1,2$ eigenmodes (Appourchaux et al. 2008; Benomar et al. 2009; Kallinger et al. 2010).

The interpretation of these eigenmodes to derive constraints on the stellar interior depends on the accuracy of the determination of the fundamental parameters of the $\operatorname{star}\left(T_{\text {eff }}, \log g,[\mathrm{Fe} / \mathrm{H}]\right)$. All chemical abundance analyses of HD 49933 converge to that of a slightly metal-poor star with $[\mathrm{Fe} / \mathrm{H}]$ between -0.3 and -0.5 (e.g. Gillon \& Magain 2006; Bruntt 2009). The determination of its effective temperature and gravity have been revisited several times. As reported in Bruntt (2009), the $T_{\text {eff }}$ obtained by different methods ranges from 6450 to $6780 \mathrm{~K}$, which is by far too uncertain to tightly constrain the stellar evolution models in the HR diagram. Similarly, $\log g$ is also not very well constrained with values ranging from 4.0 to 4.3. Several authors have attempted to determine $T_{\text {eff }}$ and $\log g$ by fitting the $\mathrm{H}_{\beta}$ and $\mathrm{Mg}$ I lines, respectively (Ryabchikova et al. 2009; Kallinger et al. 2010). Their solutions are most consistent with a cooler temperature $(\approx 6500 \mathrm{~K})$ and a lower gravity $(\approx 4.0)$.

Since spectrometry and photometry lead to stellar parameters with a large uncertainty, in this work we use a different approach by determining its diameter using interferometry. Combined with seismology, it provides a very good constraint of the stellar parameter space (Creevey et al. 2007). This approach was proposed for the first time by Kervella et al. (2003) for the binary star $\alpha$ Cen A\&B and later with similar success for other asteroseismic targets (Cunha et al. 2007; Teixeira et al. 2009; Bruntt et al. 2009; Mazumdar et al. 2009; Bazot et al. 2011).

In this letter we present the first campaign of interferometric measurements of HD 49933 obtained using the VEGA instrument (Mourard et al. 2009, 2011) at the CHARA Array (ten Brummelaar et al. 2005). We used state-of-art 3D hydrodynamical simulations of the surface of the star to calculate the limb darkening and to compute the visibility curves prior to ex- 


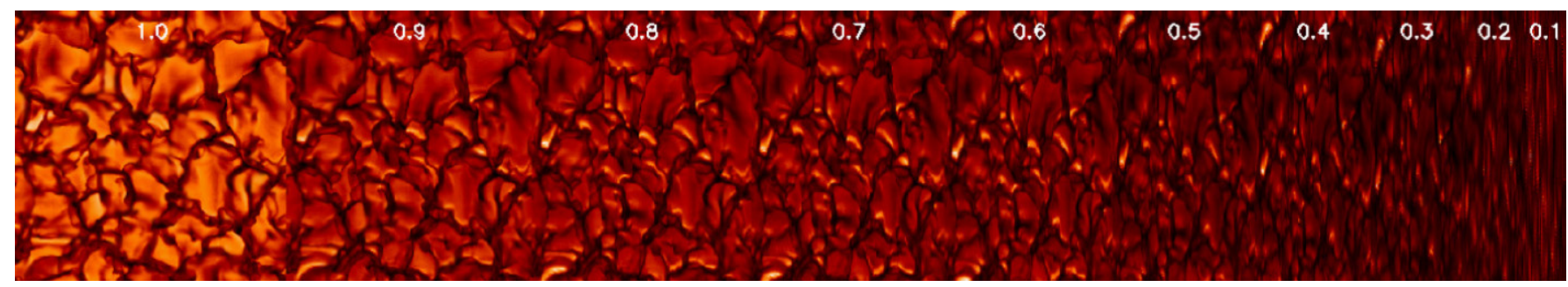

Fig. 1. Monochromatic $(730 \mathrm{~nm})$ center-to-limb emergent intensity at a representative time of the simulation for various cosines of the viewing angle: $\mu=1.0$ (disk center) down to 0.1 (towards the limb). The horizontal sizes of the simulation domain are $21000 \times 21000 \mathrm{~km}$.

tracting the angular diameter. The derived stellar radius is then used to constrain the other fundamental stellar parameters by fitting the oscillation frequency separations.

\section{CHARA/VEGA observations}

Observations of HD 49933 were performed on October 16, 2010 using the VEGA instrument of the CHARA Array and the instrument CLIMB (Sturmann et al. 2010) for 3T group delay tracking. The telescopes E1, E2, and W2 were used giving access to ground baselines of 66,156 , and $221 \mathrm{~m}$. The seeing was stable with a value of $\mathrm{r} 0$ between 10 and $13 \mathrm{~cm}$. For the absolute calibration of the squared visibilities, we observed two different calibrators, $\mathrm{C} 1=\mathrm{HD} 55185$ and C2 = HD 46487. The observing sequence was C1-T-C2-T-C2-T-C1, each block being almost $20 \mathrm{mn}$. The data were processed using the standard $V^{2}$ procedure of the VEGA instrument as described in Mourard et al. (2011). To validate the absolute calibration, we first considered $\mathrm{C} 1$ as a target and thus estimated its uniform disk (UD) diameter using $\mathrm{C} 2$ as the calibrator. A spectral band of $\Delta \lambda=30 \mathrm{~nm}$ centered around $\lambda=735 \mathrm{~nm}$ was used for the data processing. The equivalent UD diameter of $\mathrm{C} 2$ is taken as $\theta_{\mathrm{HD}} 46487=0.180 \pm 0.013$ mas according to the SearchCal tool ${ }^{1}$ of the JMMC (Bonneau et al. 2006). Fitting a UD model to the three squared visibilities provides an estimate of the angular diameter of HD 55185 of $\theta_{\mathrm{HD}} 55185=0.474 \pm 0.014$ mas. Spectral bandwidth and baseline smearing are included but only introduce a bias less than $0.1 \%$ on the diameter determination. Surface brightness relations, as provided by SearchCal, provide an independent estimate of the angular diameter. We found 0.478 mas and 0.476 mas from the $(V-R)$ and $(B-V)$ relations, respectively. Our result agrees very well with these photometric estimations. We finally use $\mathrm{C} 1$ and $\mathrm{C} 2$ to calibrate the measurements on the target. To avoid a strong telluric feature, data are processed using a spectral band of $\Delta \lambda=20 \mathrm{~nm}$ centered around $\lambda=740 \mathrm{~nm}$. The final error bars in the target measurements take into account the measurement noise, the dispersion in the individual measurements, the diameter of the calibrators, and their errors.

\section{Determination of the diameter}

To derive a reliable diameter of HD 49933, we base our analysis on a 3D hydrodynamical simulation of the surface of the star. Allende Prieto et al. (2002) demonstrated that for Procyon, a star similar to HD 49933, the 3D limb darkening is significantly different from the corresponding 1D hydrostatic and homogeneous one. The differences were found to be larger in the visible (the VEGA domain) than the infrared. We adopt the same

\footnotetext{
1 Available at http://www. jmmc . fr/searchcal
}

methodology as used for both $\alpha$ Cen B (Bigot et al. 2006) and Procyon (Aufdenberg et al. 2005). A hydrodynamical simulation of the surface is used to obtain 3D time-dependent limbdarkened intensities, instead of the simplified 1D homogeneous or UD models, to compute the visibility curves. The angular diameter is found by minimizing the differences between the synthetic and observed visibility curves.

\subsection{The 3D simulation of the surface}

We use the state-of-the-art radiative hydrodynamical code (STAGGER CODE, Nordlund \& Galsgaard ${ }^{2}$ ) to simulate the surface convection and stratification of HD 49933. In a local box, the code solves the full set of conservative hydrodynamical equations coupled to an accurate treatment of the radiative transfer. The code is based on a sixth order explicit finite difference scheme. The equations are solved on a staggered mesh where the thermodynamical variables are cell centered, while the fluxes are shifted to the cell edge. The domain of simulation contains the entropy minimum located at the surface and is extended deep enough to have a flat entropy profile at the bottom (adiabatic regime). The code uses periodic boundary conditions horizontally and open boundaries vertically. At the bottom of the simulation, the inflows have constant entropy and pressure. The outflows are not constrained and are free to pass through the boundary. We used a realistic equation-of-state that accounts for ionization, recombination, and dissociation (Mihalas et al. 1988) and continuous + line opacities (Gustafsson et al. 2008). Radiative transfer is crucial since it drives convection through entropy losses at the surface (Stein \& Nordlund 1998) and is solved using the Feautrier's scheme along several inclined rays (one vertical, eight inclined) through each grid point. The wavelength dependence of the radiative transfer is taken into account using a binning scheme in which the monochromatic lines are collected into 12 bins. The numerical resolution used for the present simulation is $240^{3}$. The geometrical sizes are $21 \times 21 \mathrm{Mm}$ horizontally and $5 \mathrm{Mm}$ vertically. The horizontal sizes of the domain are defined to contain a sufficient number of granules at each time-step and the vertical one to be deep enough to ensure an adiabatic regime at the bottom. The stellar parameters that define our 3D model are $T_{\text {eff }}=6690 \pm 35 \mathrm{~K}$, $\log g=4.21$ and a scaled solar chemical composition (Asplund et al. 2009) down to -0.5 dex. The uncertainty in $T_{\text {eff }}$ represents the fluctuations with time around the mean value. The simulation was run for a couple of stellar hours to get the full hydrodynamical relaxation. The convection in such an F star is more efficient than for the Sun: the rms vertical velocity is $3.9 \mathrm{~km} \mathrm{~s}^{-1}$, which is about twice the solar value. The granulation pattern is shown in Fig. 1.

2 1995, http://www.astro.ku.dk/ kg/Papers/MHDcode.ps.gz 


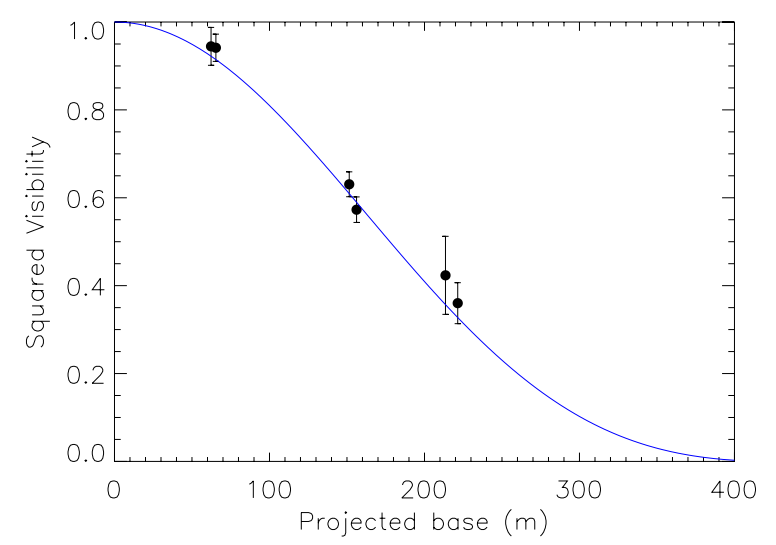

Fig. 2. Our best-fit model of the observed squared visibilities (black dots) with the calculated one (full line) with a reduced $\chi^{2}=0.47$. The angular diameter is $\theta_{\mathrm{LD}}=0.445$ mas.

\subsection{Limb darkening and visibility curves}

Hereafter, we use the methodology presented in Bigot et al. (2006). The snapshots are taken after relaxation and cover a sequence of several convective turnover times. We extract snapshots every five minutes over a sequence of one hour. For each snapshot, we calculate the radiative transfer in the selected wavelength bandwidth of the VEGA instrument $\Delta \lambda=[730-750] \mathrm{nm}$ to get the monochromatic limb-darkened intensities for each time step, each position at the stellar surface, and each cosine $\mu$ of the angle made between the vertical and the line of sight.

The normalized fringe visibilities are obtained using the Cittert-Zernike theorem

$V_{\lambda}(B, \theta)=\frac{\int_{0}^{1}\left\langle I_{\lambda}(\mu)\right\rangle J_{0}\left(\pi B \theta \lambda^{-1} \sqrt{1-\mu^{2}}\right) \mu \mathrm{d} \mu}{\int_{0}^{1}\left\langle I_{\lambda}(\mu)\right\rangle \mu \mathrm{d} \mu}$,

where $B$ is the projected baseline (in meters), $J_{0}$ is the zero order of the Bessel function, and $\left\langle I_{\lambda}(\mu)\right\rangle$ is the time- and horizontalaverage of the $3 \mathrm{D}$ intensities. We integrate the monochromatic visibilities over the spectral domain $\Delta \lambda$ of the instrument weighted by the spectral transmission $T(\lambda)$

$V^{2}(B, \theta)=\frac{\int_{\Delta \lambda}\left[V_{\lambda}(B, \theta) T(\lambda)\right]^{2} \mathrm{~d} \lambda}{\int_{\Delta \lambda}[T(\lambda)]^{2} \mathrm{~d} \lambda}$.

The function $T(\lambda)$ is derived from the direct flux measurement of the target and checked on several bright stars.

\subsection{The $\chi^{2}$ fit of the data}

The angular diameter is determined by a Levenberg-Marquardt least-squares fit to the observed squared visibilities with the model. The result is shown in Fig. 2. The value derived from the 3D limb darkening is $\theta_{\mathrm{LD}}=0.445 \pm 0.012$ mas. The reduced $\chi^{2}$ of the fit is 0.47 . Using the revised Hipparcos parallax $\pi=33.69 \pm 0.42$ mas (van Leeuwen 2007), we obtain a linear radius of $R=1.42 \pm 0.04 R_{\odot}$. The corresponding radius derived from 1D hydrostatic limb-darkening (Claret 2000) is $R=1.45 \pm 0.04 R_{\odot}$, which is significantly larger by $1 \sigma$ because of the more pronounced limb darkening in $1 \mathrm{D}$. We note that the derived radius is not very sensitive to the choice of stellar parameters (those selected in Sect. 3.1). We indeed tried several values of $T_{\text {eff }}(=6500,6750 \mathrm{~K})$ and $\log g(=4.0,4.5)$ and found very small differences in terms of derived radius $\left(\leq 0.002 R_{\odot}\right)$.
The derived angular diameter $\theta_{\mathrm{LD}}$ agrees with the prediction of $0.452 \pm 0.007$ mas obtained by the surface brightness relation (Thévenin et al. 2006). The relative uncertainty in the angular diameter $(2.7 \%)$ is larger by an order of magnitude than the smallest uncertainty ever achieved for an interferometric radius, $(0.2 \%)$ derived in the case of the $\alpha$ Centauri A (Kervella et al. 2003). However, we emphasize that the target in the present work is 20 times smaller. This uncertainty dominates the total uncertainty of about $3 \%$, including the error in the parallax. Nonetheless, the relative uncertainty in the radius is sufficiently accurate to tightly constrain the mass using asteroseismic frequencies (Creevey et al. 2007).

\section{Asteroseismic fits of acoustic frequencies}

We estimate the mass and the age of HD 49933 using asteroseismic data. The aim is to find a stellar evolutionary model whose radius equals the interferometric one, whose $T_{\text {eff }}, \log g$, and $[\mathrm{Fe} / \mathrm{H}]$ match the spectrometric ones (Bruntt 2009), and whose eigenmode frequency separations match the CoRoT data (Benomar et al. 2009). We compute a grid of models of mass between 1.7 and $2.1 M_{\odot}$, and search for the one that most closely matches the observed small and large frequency separations. The stellar model evolves until the radius equals the interferometric one. The initial metallicity is adjusted to match the spectroscopic $[\mathrm{Fe} / \mathrm{H}]$. The stellar evolutionary models are computed using the CESAM2k code (Morel 1997; Morel \& Lebreton 2008). In addition, we adopt Chemical diffusion (Burgers 1969) combined with extra-mixing (Morel \& Thévenin 2002) and the Canuto \& Mazzitelli (1991) formalism of convection. The rotation of HD 49933 has a minor impact on the modeling (Piau et al. 2009) and is ignored. For each stellar model, we compute eigenfrequencies between 1100 and $2500 \mu \mathrm{Hz}$ for different Legendre polynomial degrees $\ell=0,1,2$ by solving ${ }^{3}$ the standard linearized equations of continuity and momentum for adiabatic oscillations (see e.g. Unno et al. 1989). Since these modes pulsate in high overtones $(n)$, we added the shift in frequency caused by the centrifugal distortion of the star (Dziembowski \& Goode 1992).

We fit both the large and small frequency separations that we define as follows

$\Delta_{n, \ell}=\frac{1}{2}\left(v_{n+1, \ell}-v_{n-1, \ell}\right)$ and $\delta_{n, \ell}=v_{n, \ell}-\frac{1}{2}\left(v_{n+1, \ell}+v_{n, \ell+1}\right)$.

Since the radius is given, the large separations are mainly sensitive to the mass of the star. The small separations are more sensitive to the deep interior and are therefore a good indicator of the stellar age (e.g. Roxburgh \& Vorontsov 2003).

The solution that minimizes the differences between the observed and computed separations is given in Table 1 and the corresponding frequency separations are shown in Fig. 3. We note that a core overshoot of 0.35 is needed to fit the small separations. We get a better fit of the small separations by including the centrifugal effects on the oscillations. The fits to both small and large separations are very satisfactory and better than in Benomar et al. (2010). To roughly estimate the errors in the mass and $T_{\text {eff }}$, we use the observed errors in the average large separation $\left(\sim M^{1 / 2} R^{-3 / 2}\right)$, in the luminosity $L\left(\sim R^{2} T_{\mathrm{eff}}^{4}\right)$, and in the interferometric radius. Our stellar evolution model agrees well with one of the solutions of Creevey \& Bazot (2011), who used a local minimization method based on radial modes. The mass $\left(1.20 \pm 0.08 M_{\odot}\right)$ is close to that of other works

${ }^{3}$ Code kindly provided by W. Dziembowski. 
Table 1. Our stellar evolution model for HD 49933.

\begin{tabular}{cccccccccccccc}
\hline \hline$M / M_{\odot}$ & $R / R_{\odot}$ & $\log g$ & $Y_{0}$ & $(Z / X)_{0}$ & $\alpha$ & $\alpha_{\mathrm{ov}}$ & $\operatorname{Age}(\mathrm{My})$ & $T_{\mathrm{eff}}(\mathrm{K})$ & $\log L / L_{\odot}$ & $X_{\mathrm{c}}$ & $Y_{\mathrm{s}}$ & $(Z / X)_{\mathrm{s}}$ & {$[\mathrm{Fe} / \mathrm{H}]$} \\
\hline 1.200 & 1.42 & 4.21 & 0.29 & 0.016 & 1.00 & 0.35 & 2690 & 6640 & 0.55 & 0.47 & 0.20 & 0.011 & -0.38 \\
\hline
\end{tabular}

Notes. The mass $M$, initial helium content $Y_{0}$, metallicity $(Z / X)_{0}$, core overshoot $\alpha_{\mathrm{ov}}$, and mixing length $\alpha$ are adjusted to reproduce the radius obtained by interferometry and the observed large and small separations.
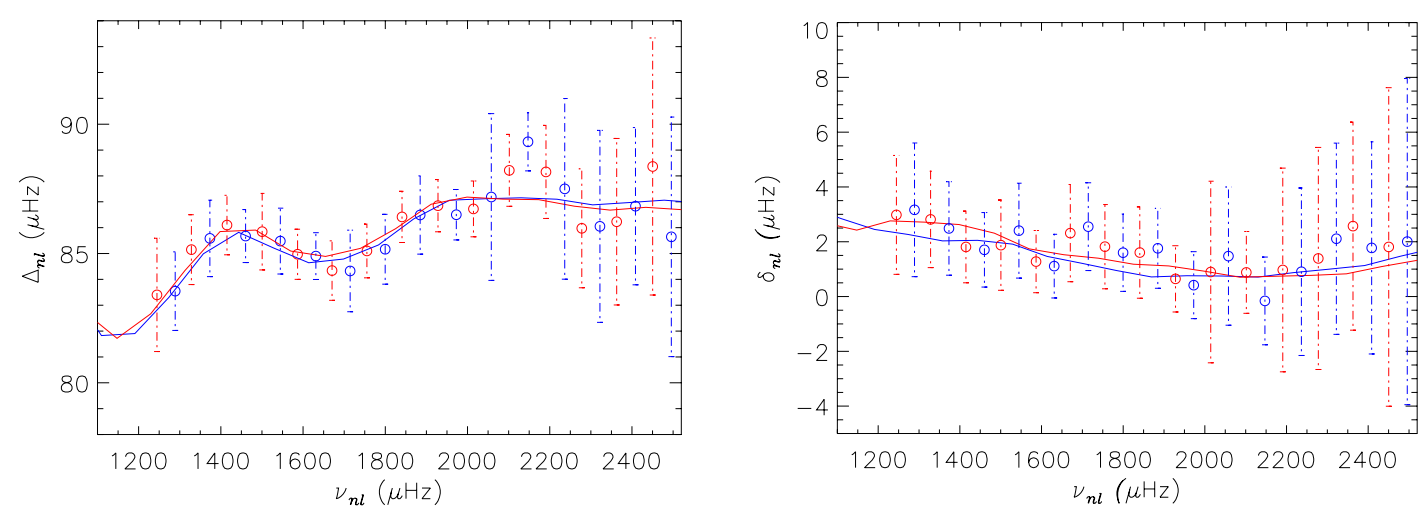

Fig. 3. Large (left panel) and small (right panel) separations for $\ell=0$ (blue) and $\ell=1$ (red) calculated with the stellar model of Table 1 . The observed data (bullets) are from Benomar et al. (2009). For each frequency, we took the maximum error bar at $1 \sigma$.

(Appourchaux et al. 2008; Benomar et al. 2010; Kallinger et al. 2010). The luminosity $\log L / L_{\odot}=0.55$ matches the one observed (Michel et al. 2008). The derived atmospheric parameters $\left(T_{\text {eff }}=6640 \pm 100 \mathrm{~K}, \log g=4.21 \pm 0.14,[\mathrm{Fe} / \mathrm{H}]=-0.38\right)$ are in good agreement with the values obtained by Strömgren photometry (Bruntt 2009). Our $T_{\text {eff }}$ is larger than the value $(\approx 6500 \mathrm{~K})$ derived from $\mathrm{H}_{\beta}$ (Ryabchikova et al. 2009; Kallinger et al. 2010). However, we emphasize that this strong line is not an excellent temperature indicator since it spreads over a wide wavelength range.

\section{Conclusion}

We have reported our long-baseline interferometric observations of the CoRoT target HD 49933 using the VEGA instrument at the CHARA Array. Combined with 3D hydrodynamical simulations of the surface, we derived its radius, $R=1.42 \pm 0.04 R_{\odot}$, which agrees with the radius derived from the surface brightness relation. The use of this interferometric radius reduces the stellar parameter space and helped us to more tightly constrain the position of the star in the HR diagram. The fit of the p-mode frequencies led us to determine the other stellar parameters. The derived atmospheric ones $\left(T_{\text {eff }}=6640 \pm 100 \mathrm{~K}, \log g=4.21 \pm 0.14\right.$, $[\mathrm{Fe} / \mathrm{H}]=-0.38$ ) agree well with photometric studies.

Acknowledgements. L.B. thanks A. Chiavassa, R. Collet, and R. Trampedach who provided the latest EOS and opacities for the $3 \mathrm{D}$ modeling, and $\mathrm{J}$. Provost for valuable discussions about the fitting of frequencies and the stellar evolution modeling. Computations have been done on the "Mesocentre SIGAMM" machine, hosted by the Observatoire de la Côte d'Azur. The CHARA Array is operated with support from the National Science Foundation through grant AST-0908253, from Georgia State University, the W. M. Keck Foundation, and the NASA Exoplanet Science Institute.

\section{References}

Allende Prieto, C., Asplund, M., García López, R. J., \& Lambert, D. L. 2002, ApJ, 567, 544

Appourchaux, T., Michel, E., Auvergne, M., et al. 2008, A\&A, 488, 705

Asplund, M., Grevesse, N., Sauval, A. J., \& Scott, P. 2009, ARA\&A, 47, 481
Aufdenberg, J. P., Ludwig, H., \& Kervella, P. 2005, ApJ, 633, 424

Baglin, A., Auvergne, M., Barge, P., et al. 2006, in ESA SP 1306, ed. M. Fridlund, A. Baglin, J. Lochard, \& L. Conroy, 33

Bazot, M., Ireland, M. J., Huber, D., et al. 2011, A\&A, 526, L4

Benomar, O., Baudin, F., Campante, T. L., et al. 2009, A\&A, 507, L13

Benomar, O., Baudin, F., Marques, J. P., et al. 2010, Astron. Nachr., 331, 956

Bigot, L., Kervella, P., Thévenin, F., \& Ségransan, D. 2006, A\&A, 446, 635

Bonneau, D., Clausse, J., Delfosse, X., et al. 2006, A\&A, 456, 789

Bruntt, H. 2009, A\&A, 506, 235

Bruntt, H., Kurtz, D. W., Cunha, M. S., et al. 2009, MNRAS, 396, 1189

Burgers, J. M. 1969, Flow Equations for Composite Gases (New York: Academic Press)

Canuto, V. M., \& Mazzitelli, I. 1991, ApJ, 370, 295

Claret, A. 2000, A\&A, 363, 1081

Creevey, O. L., \& Bazot, M. 2011, J. Phys. Conf. Ser., 271, 012038

Creevey, O. L., Monteiro, M. J. P. F. G., Metcalfe, T. S., et al. 2007, ApJ, 659, 616

Cunha, M. S., Aerts, C., Christensen-Dalsgaard, J., et al. 2007, A\&AR, 14, 217

Dziembowski, W. A., \& Goode, P. R. 1992, ApJ, 394, 670

Gillon, M., \& Magain, P. 2006, A\&A, 448, 341

Gustafsson, B., Edvardsson, B., Eriksson, K., et al. 2008, A\&A, 486, 951

Kallinger, T., Gruberbauer, M., Guenther, D. B., Fossati, L., \& Weiss, W. W. 2010, A\&A, 510, A106

Kervella, P., Thévenin, F., Ségransan, D., et al. 2003, A\&A, 404, 1087

Mazumdar, A., Mérand, A., Demarque, P., et al. 2009, A\&A, 503, 521

Michel, E., Baglin, A., Auvergne, M., et al. 2008, Science, 322, 558

Mihalas, D., Dappen, W., \& Hummer, D. G. 1988, ApJ, 331, 815

Morel, P. 1997, A\&AS, 124, 597

Morel, P., \& Lebreton, Y. 2008, Ap\&SS, 316, 61

Morel, P., \& Thévenin, F. 2002, A\&A, 390, 611

Mosser, B., Bouchy, F., Catala, C., et al. 2005, A\&A, 431, L13

Mourard, D., Clausse, J. M., Marcotto, A., et al. 2009, A\&A, 508, 1073

Mourard, D., Bério, P., Perraut, K., et al. 2011, A\&A, 531, A110

Piau, L., Turck-Chièze, S., Duez, V., \& Stein, R. F. 2009, A\&A, 506, 175

Roxburgh, I. W., \& Vorontsov, S. V. 2003, A\&A, 411, 215

Ryabchikova, T., Fossati, L., \& Shulyak, D. 2009, A\&A, 506, 203

Stein, R. F., \& Nordlund, A. 1998, ApJ, 499, 914

Sturmann, J., Ten Brummelaar, T., Sturmann, L., \& McAlister, H. A. 2010, in SPIE Conf. Ser. 7734, ed. W. Danchi, C. William, F. Delplancke, \& J. K. Rajagopal

Teixeira, T. C., Kjeldsen, H., Bedding, T. R., et al. 2009, A\&A, 494, 237

ten Brummelaar, T. A., McAlister, H. A., Ridgway, S. T., et al. 2005, ApJ, 628, 453

Thévenin, F., Bigot, L., Kervella, P., et al. 2006, Mem. Soc. Astron. It., 77, 411

Unno, W., Osaki, Y., Ando, H., Saio, H., \& Shibahashi, H. 1989, Nonradial oscillations of stars, 2nd ed. (Tokyo: University of Tokyo Press)

van Leeuwen, F. 2007, A\&A, 474, 653 